\title{
Precision metrology of weak measurement with thermal state pointer
}

\author{
Gang Li* ${ }^{1}{ }_{\text {Li-Bo Chen }}{ }^{2}$ Tao Wang ${ }^{\dagger},{ }^{3}$ Zhi-Hui He, ${ }^{1}$ and He-Shan $\mathrm{Song}^{4}$ \\ ${ }^{1}$ School of Physics and Electronic Information, Yan'an University, Yanan 716000, China \\ ${ }^{2}$ School of Science, Qingdao Technological University, Qingdao 266033, China \\ ${ }^{3}$ College of Physics, Tonghua Normal University, Tonghua 134000, China and \\ ${ }^{4}$ School of Physics, Dalian University Of Technology, Dalian 116024, China
}

(Dated: November 13, 2018)

\begin{abstract}
Quantum metrology is being gradually studied for weak measurement systems. For weak measurement systems with thermal state pointer, we find that in the displacement space corresponding to imaginary weak values, the maximal QFI after successful postselection can attain the level of thermal fluctuations, without surpassing total QFI, and that QFI which increases with increasing temperature can constantly improve the measurement precision. These results are much better than that of weak measurement with pure state (i.e., Gaussian state) pointer. On the other hand, in Kerr nonlinear interaction systems with weak measurement, and by using thermal state pointer, we obtain in the phase space successful postselection and postselected measurements both achieve the Heisenberg limit of quantum metrology, and show weak measurement with thermal states only obtain classical Fisher information (CFI) which increases with increasing temperature and achieves classical enhanced scaling of $N^{2}$. Moreover, weak measurement with thermal states has an advantage over that with coherent states or mixed states of the light because generating these states with more large uncertainty are limited under the current technology, but thermal states with more large uncertainly are very easy to achieve with increasing temperature in nature, regardless of thermal states of the light or the matter.
\end{abstract}

PACS numbers: 42.50.Wk, 42.65.Hw, 03.65.Ta

\section{INTRODUCTION}

Quantum metrology is committed to enhancing measurement precision and developing measurement techniques that give better precision than the same measurement performed in a classical framework, therefore, it has aroused substantial interest owing to its vital importance in physics and other sciences [1 10]. By now, for the estimation of a parameter $\chi$ with a pointer state that contains on average $N$ particles, a major way to improving measurement precision is by utilizing non-classical resources, such as quantum entanglement $11-13]$ and squeezed states [14 17], which indicate that an improved measurement precision can surpass the standard quantum limit $(\delta \chi \propto 1 / \sqrt{N})$ or even achieves the Heisenberg scaling $(\delta \chi \propto 1 / N)$. However, the difficulty in generating highly entangled states and fragility of such states is the open challenge to enhance measurement precision beyond classical techniques in practical applications. Moreover, whether quantum resource is essential for quantum enhanced precision. Recently, by considering photon coupling with coherent state pointer, the quantum Fisher information (QFI) can show a quantum scaling of $N^{2}$ 18], even without any quantum resources, and soon it was experimentally verified via coherent light as a pointer [19]. Meanwhile, for the mixed states with modulating the power of coherent light, measurement precision can

\footnotetext{
*ligang0311@sina.cn

†suiyueqiaoqiao@163.com
}

experimentally attain Heisenberg scaling in weak measurement 20]. Thermal states are real classical states in nature, but they are not considered as the pointers in the original weak measurement protocol because their thermal fluctuations increase with temperature [21]. Therefore, in most weak measurement studies, the pure states (i.e., Gaussian states) are generally considered to be the pointers. However, we have shown that by using thermal states as the pointers can amplify weak measurement effect [22]. These results motivate us to pursue innovative precision metrology schemes.

In this paper, we study quantum metrology of thermal states based on weak measurement to bridge this gap. We show that in the displacement space corresponding to imaginary weak values, at weak measurement limit the maximal QFI after successful post-selection can attain the level of thermal fluctuations, without surpassing total QFI, that weak measurement with thermal states offer better precision relative to weak measurement with pure states (i.e., Gaussian states), and that as the temperature increases, QFI is also increased, thereby constantly improving the precision of parameter estimation. On the other hand, for Kerr nonlinear interaction systems with weak measurement, we surprisingly find that in the phase space successful postselection and postselected measurement can beat the standard quantum limit and achieve the Heisenberg limit of quantum metrology using classical resources, i.e., thermal states, that it is shown that weak measurement with thermal states only obtain classical Fisher information (CFI) increasing when temperature increases, and it has an advantage over weak 
measurement with coherent states or mixed states of the light because generating these states with large uncertainty are limited under current technology, but thermal states with large uncertainly are very easy to achieve with increasing temperature in nature.

The structure of our paper is as follows. In Sec. II, we give a discussion of quantum metrology of weak measurement with a thermal state pointer, including precision measurement in the displacement space and the phase space. In Sec. III, we obtain the conclusion of the work.

\section{QUANTUM METROLOGY OF WEAK MEASUREMENT WITH THERMAL STATE POINTER}

\section{Precision measurement in the displacement space}

\section{Weak measurement amplification model}

In the standard scenario of weak measurement 21 26], the interaction Hamiltonian between the system and the pointer is (assuming $\hbar=1$ )

$$
\hat{H}=\hbar \chi(t) \hat{A} \otimes \hat{q},
$$

where $A$ is a system observable, $q$ is the position observable of the pointer and $A$ to be measured is usually a twolevel system and the pointer is a continuous system. $\chi(t)$ is a narrow pulse function with interaction strengrh $\chi$. Suppose the initial system state is $\left|\psi_{i}\right\rangle=\sum_{i=1}^{2} c_{i}\left|a_{i}\right\rangle$ with $A\left|a_{i}\right\rangle=a_{i}\left|a_{i}\right\rangle$, where $c_{1}=\cos \frac{\theta_{i}}{2}$ and $c_{2}=e^{i \varphi} \sin \frac{\theta_{i}}{2}$ with $0 \leq \theta_{i} \leq \pi$. Then we consider the initial pointer state as $\rho_{t h}(z)=(1-z) \sum_{n=0}^{\infty} z^{n}|n\rangle_{m}\left\langle\left. n\right|_{m}\right.$ with $z=e^{-\hbar \omega_{m} / k_{B} T}$, where $k_{B}$ is the Boltzmann constant and $T$ is the temperature.

The evolution of the total system after an interaction $U(t)=e^{-i \chi A q}$ is given by

$$
\rho(z)=e^{-i \chi A q}\left|\psi_{i}\right\rangle\left\langle\psi_{i}\right| \otimes \rho_{t h}(z) e^{i \chi A q} .
$$

For Eq. (2), in momentum $p$ coordinate space we have

$$
\begin{aligned}
\rho^{p}(z) & =\sum_{i, j=1, n=0}^{2, \infty} \iint_{-\infty}^{\infty} \frac{(1-z) z^{n}}{2^{n} n !} c_{i} c_{j}^{*}\left|a_{i}\right\rangle\left\langle a_{j}\right| G_{n}\left(p_{i}\right) \\
& \times G_{n}\left(p_{j}^{\prime}\right) d p d p^{\prime}|p\rangle\left\langle p^{\prime}\right|,
\end{aligned}
$$

$$
G_{n}\left(p_{i}\right)=H_{n}\left(\sqrt{2} \sigma p_{i}\right) \phi_{0}\left(p_{i}\right),
$$

where $\phi_{0}\left(p_{i}\right)=\left(\frac{2 \pi}{4 \sigma^{2}}\right)^{-1 / 4} \exp \left(-\sigma^{2} p_{i}^{2}\right)$ with $p_{i}=p+a_{i} \chi$ and $\sigma$ is zero point fluctuation, and $H_{n}$ is Hermite Polynomial.

Under this dynamics, each of the eigenstates $\left|a_{i}\right\rangle$ of the system observable $A$ is entangled with the pointer state wavefunctions, which is translated by the different $a_{i} \chi$ proportional to the eigenvalue $a_{i}$. When $\left|a_{i}-a_{j}\right| \chi$ is much larger than the width $\sqrt{\frac{1+z}{1-z}} \frac{1}{2 \sigma}$ of $\rho_{t h}(z)$, this becomes a strong measurement, meaning that the overlap

$$
\begin{aligned}
O_{i j}:= & \left(\frac{1+z}{1-z}\right)^{-1 / 2} \sum_{i \neq j} \int \exp \left[\left(4 \sigma^{2} p_{i} p_{j} z-2 \sigma^{2}\left(p_{i}^{2}\right.\right.\right. \\
+ & \left.\left.\left.p_{j}^{2}\right) z^{2}\right) /\left(1-z^{2}\right)\right] \phi_{0}\left(p_{i}\right) \phi_{0}\left(p_{j}\right) d p
\end{aligned}
$$

between each pair of shifted wavefunctions is vanishingly small. So the pointer state corresponding to different eigenvalues becomes completely separated. However, when $\left|a_{i}-a_{j}\right| \chi$ is relatively small and the wavefunctions are no longer well resolved, the measurement is said to be weak [27].

When the postselected state of the measured system $\left|\psi_{a}\right\rangle=\sum_{i=1}^{2} c_{i}^{\prime}\left|a_{i}\right\rangle$ with $c_{1}^{\prime}=\cos \frac{\theta_{f}}{2}$ and $c_{2}^{\prime}=-\sin \frac{\theta_{f}}{2}(0 \leq$ $\left.\theta_{f} \leq \pi\right)$ is performed for the total system (3), then $\rho^{p}(z)$ reduces to

$$
\begin{aligned}
\rho^{p}(z) & =\sum_{i, j=1, n=0}^{2, \infty} \iint_{-\infty}^{\infty} c_{i} c_{i}^{\prime *} c_{j}^{\prime} c_{j}^{*} \frac{(1-z) z^{n}}{2^{n} n !} G_{n}\left(p_{i}\right) \\
& \times G_{n}^{*}\left(p_{j}^{\prime}\right) d p d p^{\prime}|p\rangle\left\langle p^{\prime}\right| .
\end{aligned}
$$

After measuring the pointer in the $p$ basis, and using identity $\psi(p)=\int_{-\infty}^{\infty} \delta\left(p-p^{\prime}\right) \psi\left(p^{\prime}\right) d p^{\prime}$, and Mehler's Hermite Polynomial Formula $\sum_{n=0}^{\infty} \frac{H_{n}(x) H_{n}(y)}{n !}\left(\frac{w}{2}\right)^{n}=(1-$ $\left.w^{2}\right)^{-1 / 2} \exp \left[\frac{2 x y w-\left(x^{2}+y^{2}\right) w^{2}}{1-w^{2}}\right]$, the probability distribution of Eq. (6) over $p$ becomes

$$
\begin{aligned}
P_{w m}(p) & =\frac{1}{P_{a}}\left(2 \pi \nabla^{2}\right)^{-1 / 2}\left[r^{2} \exp \left[-\frac{\left(p+a_{1} \chi\right)^{2}}{2 \nabla^{2}}\right]\right. \\
& -2 r t\left(e^{-i \varphi}+e^{i \varphi}\right) \exp \left[-\frac{\left(p+\frac{a_{2}-z a_{1}}{1-z}\right)^{2}}{4 \nabla^{2}}\right. \\
& \left.-\frac{\left(p+\frac{a_{1}-z a_{2}}{1-z}\right)^{2}}{4 \nabla^{2}}\right]+t^{2} \exp \left[-\frac{\left(p+a_{2} \chi\right)^{2}}{2 \nabla^{2}}\right],
\end{aligned}
$$

where $r=\cos \frac{\theta_{i}}{2} \cos \frac{\theta_{f}}{2}, t=\sin \frac{\theta_{i}}{2} \sin \frac{\theta_{f}}{2}, \nabla^{2}=\frac{1+z}{1-z} \frac{1}{4 \sigma^{2}}$, and the probability of successful postselection is

$$
P_{a}=r^{2}+t^{2}-2 r t \exp \left[-\frac{\left(a_{1}-a_{2}\right)^{2} \chi^{2} \Delta^{2}}{2}\right] \cos \varphi,
$$

where $\Delta^{2}=\frac{1+z}{1-z} \sigma^{2}$.

After postselection measurement, in position $q$ coordinate space the reduced state of Eq. (2) is given by

$$
\begin{gathered}
\rho^{q}(z)=\sum_{i, j=1, n=0}^{2, \infty} \iint_{-\infty}^{\infty} c_{i} c_{i}^{\prime *} c_{j}^{\prime} c_{j}^{*} \frac{(1-z) z^{n}}{2^{n} n !} G_{n}(q) \\
\times G_{n}\left(q^{\prime}\right) e^{-i \chi q\left(a_{i}-a_{j}\right)} d q d q^{\prime}|q\rangle\left\langle q^{\prime}\right|, \\
G_{n}(q)=H_{n}\left(\frac{q}{\sqrt{2} \sigma}\right) \phi_{0}(q),
\end{gathered}
$$


where $\phi_{0}(q)=\left(2 \pi \sigma^{2}\right)^{1 / 4} \exp \left(-\frac{q^{2}}{4 \sigma^{2}}\right)$.

After measuring the pointer in $q$ basis, the probability distribution of Eq. (9) over $q$ is given by

$$
\begin{aligned}
P_{w m}(q) & =\frac{1}{P_{a}}\left(2 \pi \Delta^{2}\right)^{-1 / 2} \exp \left[-\frac{q^{2}}{2 \Delta^{2}}\right]\left[r^{2}-r t\right. \\
& \times\left(\exp \left[i \varphi-i\left(a_{2}-a_{1}\right) \chi q\right]+\exp \left[i \left(a_{2}\right.\right.\right. \\
& \left.\left.\left.\left.-a_{1}\right) \chi q-i \varphi\right]\right)+t^{2}\right] .
\end{aligned}
$$

When the postselection fails (with probability $P_{r}=$ $1-P_{a}$ ), namely, the failing postselected state of the measured system $\left|\psi_{r}\right\rangle=\sum_{i=1}^{2} c_{i}^{\prime}\left|a_{i}\right\rangle$ with $c_{1}^{\prime}=\sin \frac{\theta_{f}}{2}$ and $c_{2}^{\prime}=\cos \frac{\theta_{f}}{2}$ is performed for the total system (2), we calculate carefully and obtain the probalility distribution of failing postselection in $p$ basis

$$
\begin{aligned}
P_{w m}(p) & =\frac{1}{P_{r}}\left(2 \pi \nabla^{2}\right)^{-1 / 2}\left[r^{\prime 2} \exp \left[-\frac{\left(p+a_{1} \chi\right)^{2}}{2 \nabla^{2}}\right]\right. \\
& +2 r^{\prime} t^{\prime}\left(e^{-i \varphi}+e^{i \varphi}\right) \exp \left[-\frac{\left(p+\frac{a_{2}-z a_{1}}{1-z}\right)^{2}}{4 \nabla^{2}}\right. \\
& \left.-\frac{\left(p+\frac{a_{1}-z a_{2}}{1-z}\right)^{2}}{4 \nabla^{2}}\right]+t^{\prime 2} \exp \left[-\frac{\left(p+a_{2} \chi\right)^{2}}{2 \nabla^{2}}\right],
\end{aligned}
$$

and the probalility distribution of failing postselection in $q$ basis

$$
\begin{aligned}
P_{w m}(q) & =\frac{1}{P_{r}}\left(2 \pi \Delta^{2}\right)^{-1 / 2} \exp \left[-\frac{q^{2}}{2 \Delta^{2}}\right]\left[r^{\prime 2}+r^{\prime} t^{\prime}\right. \\
& \times\left(\exp \left[i \varphi-i\left(a_{2}-a_{1}\right) \chi q\right]+\exp \left[i \left(a_{2}\right.\right.\right. \\
& \left.\left.\left.\left.-a_{1}\right) \chi q-i \varphi\right]\right)+t^{\prime 2}\right],
\end{aligned}
$$

where $r^{\prime}=\cos \frac{\theta_{i}}{2} \sin \frac{\theta_{f}}{2}$ and $t^{\prime}=\sin \frac{\theta_{i}}{2} \cos \frac{\theta_{f}}{2}$.

\section{Metric}

In the precision metrology, a parameter estimation interested can be given by the Fisher information (FI) [28], and it is functional on such conditional probability distributions, and is defined as follows

$$
F_{\chi}[P(s \mid \chi)]=\int_{-\infty}^{\infty} \frac{\left(\partial_{x} P(s \mid \chi)\right)^{2}}{P(s \mid \chi)} d s,
$$

where $s$ represents $p$ or $q$. The sensitive estimate of an unknown parameter is given by the observed statistics [29], i.e., Cramer-Rao bound limits

$$
\operatorname{Var}(\chi) \geq \frac{1}{N F_{\chi}},
$$

where $\operatorname{Var}()$ is a variance expression and $N$ is the number of independent trials.

If the postselected weak measurement is applied to the precision metrology of a parameter estimation, the whole process is called weak measurement amplification strategy (i.e., WMA strategy). Conversely, the standard strategy is when there is no weak measurement, and it refers to the benchmark measurement strategy completely ignoring the degree of freedom of the system. For Eq. (3), one traces over the degree of freedom of the system and measures the particle in $p$ basis to give

$$
P_{\text {std }}^{j o i n t}(p, z)=\sum_{i=1}^{2}\left|c_{i}\right|^{2} \phi\left(p+a_{i} \chi\right)
$$

where $\phi\left(p+a_{i} \chi\right)=\left(2 \pi \nabla^{2}\right)^{-1 / 2} \exp \left[-\left(p+a_{i} \chi\right)^{2} /\left(2 \nabla^{2}\right)\right]$.

Substitute (16) into the FI fomula (14) and applying the Cauchy-Schwarz inequality, the quantum Fisher information (QFI) of the joint system state before postselection in the momentum $p$ coordinate space is

$$
\begin{aligned}
Q_{j}(p) & =\nabla^{-4} \int_{-\infty}^{\infty} \frac{\left[\sum_{i=1}^{2}\left|c_{i}\right|^{2} \phi\left(p+a_{i} \chi\right)\left(p+a_{i} \chi\right) a_{i}\right]^{2}}{P_{\text {std }}^{\text {joint }}(p \mid \chi)} d p \\
& \leq \nabla^{-4} \int_{-\infty}^{\infty} \sum_{i=1}^{2}\left|c_{i}\right|^{2} a_{i}^{2} \phi\left(p+a_{i} \chi\right)\left(p+a_{i} \chi\right)^{2} d p \\
& =\nabla^{-2} \sum_{i=1}^{2}\left|c_{i}\right|^{2} a_{i}^{2} \\
& \leq\left|a_{i}\right|_{\max }^{2} \nabla^{-2} .
\end{aligned}
$$

We let $F_{\chi}\left(P_{\text {std }}(p, z)\right)$ take the maximum value of (17), i.e., $F_{\chi}\left(P_{\text {std }}(p, z)\right)=\left|a_{i}\right|_{\max }^{2} \nabla^{-2}$. However, when $z=0$,

$$
F_{\chi}\left(P_{\text {std }}(p, z=0)\right)=\left|a_{i}\right|_{\max }^{2}\left(\frac{1}{4 \sigma^{2}}\right)^{-1},
$$

which shows the highest FI of pure Gaussian states in a standard strategy. It can be seen from Eq. (18) that in a standard strategy using pure Gaussian states give the higher estimate of an unkown parameter $\chi$ than using thermal states. Therefore, the use of thermal states in weak measurement has no advantage over the use of pure states in terms of conventional measurement. Here we use QFI of pure states as the benchmark in the standard strategy.

In the WMA strategy, the QFI can be divided into three parts, and $F_{Q}^{t o t}=P_{a} F_{Q}^{a}+P_{r} F_{Q}^{r}+F_{Q}^{p}$ (see QFI derivation of [18]), where $F_{Q}^{a}$ and $F_{Q}^{r}$ denote the QFI of successful postselection (accepted information) and failing postselection (rejected information) for weak measurement, respectively, and $F_{Q}^{p}$ is classical FI for projective measurement. Since $F_{Q}^{\text {tot }}(p) \leq Q_{j}(p)$ [18], and in the momentum $p$ coordinate space, $Q_{j}(p) \leq F_{\chi}\left(P_{\text {std }}(p, z) \leq\right.$ $F_{\chi}\left(P_{\text {std }}(p, z=0)\right)$. Therefore, $F_{Q}^{\text {tot }}(p) \leq Q_{j}(p) \leq$ $F_{\chi}\left(P_{\text {std }}(p, z=0)\right)$.

Substitute (11) and (13) into the FI fomula (14), re- 
spectively, in the position $q$ coordinate space,

$$
\begin{aligned}
F_{Q}^{a}(q) & =\frac{-4 r^{2} t^{2}}{P_{a}^{2}} \exp \left[-\left(a_{2}-a_{1}\right)^{2} \chi^{2} \Delta^{2}\right] \cos ^{2} \varphi \Delta^{4} \\
& \times\left(a_{2}-a_{1}\right)^{4} \chi^{2}+\frac{r^{2} t^{2}}{P_{a}}\left(a_{2}-a_{1}\right)^{2}\left(2 \pi \Delta^{2}\right)^{-1 / 2} \\
& \times \int_{-\infty}^{\infty} q^{2} \exp \left(-\frac{q^{2}}{2 \Delta^{2}}\right)\left(4-\left(\operatorname { e x p } \left[i\left(a_{2}-a_{1}\right) \chi q\right.\right.\right. \\
& \left.\left.\left.-i \varphi)]+\exp \left[i \varphi-i\left(a_{2}-a_{1}\right) \chi q\right)\right]\right)^{2}\right) d q /\left[r^{2}\right. \\
& +t^{2}-r t\left(\exp \left[i\left(a_{2}-a_{1}\right) \chi q-i \varphi\right)\right]+\exp [i \varphi \\
& \left.\left.\left.-i\left(a_{2}-a_{1}\right) \chi q\right]\right)\right],
\end{aligned}
$$

and

$$
\begin{aligned}
F_{Q}^{r}(q) & =\frac{-4 r^{2} t^{2}}{P_{r}^{2}} \exp \left[-\left(a_{2}-a_{1}\right)^{2} \chi^{2} \Delta^{2}\right] \cos ^{2} \varphi \Delta^{4} \\
& \times\left(a_{2}-a_{1}\right)^{4} \chi^{2}+\frac{r^{2} t^{2}}{P_{r}}\left(a_{2}-a_{1}\right)^{2}\left(2 \pi \Delta^{2}\right)^{-1 / 2} \\
& \times \int_{-\infty}^{\infty} q^{2} \exp \left(-\frac{q^{2}}{2 \Delta^{2}}\right)\left(4-\left(\operatorname { e x p } \left[i\left(a_{2}-a_{1}\right) \chi q\right.\right.\right. \\
& \left.\left.\left.-i \varphi)]+\exp \left[i \varphi-i\left(a_{2}-a_{1}\right) \chi q\right)\right]\right)^{2}\right) d q /\left[r^{\prime 2}\right. \\
& +t^{\prime 2}+r t\left(\exp \left[i\left(a_{2}-a_{1}\right) \chi q-i \varphi\right)\right]+\exp [i \varphi \\
& \left.\left.\left.-i\left(a_{2}-a_{1}\right) \chi q\right]\right)\right] .
\end{aligned}
$$

Moreover,

$$
\begin{aligned}
F_{Q}^{P} & =\frac{P_{a}^{\prime^{2}}}{P_{a}}+\frac{P_{r}^{\prime^{2}}}{P_{r}} \\
& =\left(\frac{4 r^{2} t^{2}}{P_{a}}+\frac{4 r^{2} t^{2}}{P_{r}}\right) \exp \left[-\left(a_{2}-a_{1}\right)^{2} \chi^{2} \Delta^{2}\right] \\
& \times \cos ^{2} \varphi \Delta^{4}\left(a_{2}-a_{1}\right)^{4} \chi^{2} .
\end{aligned}
$$

Therefore,

$$
\begin{aligned}
& F_{Q}^{t o t}(q)=r^{2} t^{2}\left(a_{2}-a_{1}\right)^{2}\left(2 \pi \Delta^{2}\right)^{-1 / 2} \int_{-\infty}^{\infty} \exp \left(-\frac{q^{2}}{2 \Delta^{2}}\right) \\
& \times q^{2}\left(4-\left(\exp \left[i\left(a_{2}-a_{1}\right) \chi q-i \varphi\right)\right]+\exp [i \varphi\right. \\
& \left.\left.\left.\left.-i\left(a_{2}-a_{1}\right) \chi q\right)\right]\right)^{2}\right) d q\left(1 /\left[r^{2}+t^{2}-r t\left(\operatorname { e x p } \left[i \left(a_{2}\right.\right.\right.\right.\right. \\
& \left.\left.\left.\left.\left.-a_{1}\right) \chi q-i \varphi\right)\right]+\exp \left[i \varphi-i\left(a_{2}-a_{1}\right) \chi q\right]\right)\right] \\
& +1 /\left[r^{\prime 2}+t^{\prime 2}+r t\left(\exp \left[i\left(a_{2}-a_{1}\right) \chi q-i \varphi\right)\right]\right. \\
& \left.\left.\left.+\exp \left[i \varphi-i\left(a_{2}-a_{1}\right) \chi q\right]\right)\right]\right) \\
& \leq 4 r t\left(a_{2}-a_{1}\right)^{2}\left(2 \pi \Delta^{2}\right)^{-1 / 2} \int_{-\infty}^{\infty} q^{2} \exp \left(-\frac{q^{2}}{2 \Delta^{2}}\right) \\
& =4 r t\left(a_{2}-a_{1}\right)^{2} \Delta^{2}
\end{aligned}
$$

and the equality holds up if and only if $\cos \frac{\theta_{i}}{2}=\sin \frac{\theta_{i}}{2}$ and $\cos \frac{\theta_{f}}{2}=\sin \frac{\theta_{f}}{2}$. Set the average number of thermal states $\langle n\rangle=\frac{z}{1-z}$, the maximal account of $F_{Q}^{\text {tot }}(q)$ can be rewritten as $\left(a_{2}-a_{1}\right)^{2}(2\langle n\rangle+1) \sigma^{2}$. Thus, the scaling is at the standard quantum limit. However, in the position $q$ coordinate space, there is no information for the joint state
(2), i.e., $Q_{j}(q)=0$. Indicate that the QFI of Eq. (19)Eq. (22) in the position space originate in postselected measurement, that is, QFI in the position space can be generated as long as projective measurement occurs.

However, our interesting attention in this paper is the Cramer-Rao bound for the WMA strategy, and how it compares to that for the standard strategy. In the limit of $N \rightarrow \infty$, their ratio is equal to $P_{a} F_{Q}^{a}(p)$ and $P_{a} F_{Q}^{a}(q)$ to $F_{\chi}\left(P_{\text {std }}(p, z=0)\right)$, respectively. The formers are corrected by the probability of successful postselection.

\section{Ideal detector}

Here we consider a stable and ideal detector (i.e., without technical imperfection). In the WMA strategy, if choosing to measure in momentum space or position space, one will obtain the displacement proportional to the real part of weak values or one proportional to the imaginary part of weak values, respectively. The corresponding conditional probability distribution is given by (77) or (11). Then taking a ratio of the WMA strategy to the standard strategy, for (7) and (18) we give

$$
\begin{aligned}
& \frac{P_{a} F_{Q}^{a}\left[P_{w m}(p)\right]}{F_{\chi}\left(P_{\text {std }}(p, z=0)\right)} \\
= & P_{a} \int_{-\infty}^{\infty} \frac{\left(\partial_{\chi} P_{w m}(p)\right)^{2}}{P_{w m}(p)} d p /\left[\left|a_{i}\right|_{\max }^{2}\left(\frac{1}{4 \sigma^{2}}\right)^{-1}\right] .
\end{aligned}
$$

The numberator of (23) is QFI for the momentum's displacement proportional to real weak values. Note that is no larger than QFI of the joint system state (6) without postselection [18], i.e., $Q_{j}(p)=\left|a_{i}\right|_{\max }^{2} \nabla^{-2}$. However, in weak measurement limit, i.e., $\chi \sigma \rightarrow 0$, $P_{a} \int_{-\infty}^{\infty} \frac{\left(\partial_{\chi} P_{w m}(p)\right)^{2}}{P_{w m}(p)} d p$ is equal to $\left(r a_{1}-t a_{2}\right)^{2} \nabla^{-2}$. Therefore, $\frac{P_{a} F_{Q}^{a}\left[P_{w m}(p)\right]}{F_{\chi}\left(P_{\text {std }}(p, z=0)\right)} \leq \frac{1-z}{1+z}$, and the equality holds up if and only if $\cos \frac{\theta_{i}}{2}=\sin \frac{\theta_{i}}{2}$ and $\cos \frac{\theta_{f}}{2}=\sin \frac{\theta_{f}}{2}$. It is clear that QFI for real weak values space using thermal state pointer can be no advantage for the purpose of estimating $\chi$.

Multiplying Eq. (8) by Eq. (19), I further give

$$
\begin{aligned}
P_{a} F_{Q}^{a}(q) & \leq \frac{-4 r^{2} t^{2} \Delta^{4}\left(a_{2}-a_{1}\right)^{4} \chi^{2}}{P_{a}} \exp \left[-\left(a_{2}\right.\right. \\
& \left.\left.-a_{1}\right)^{2} \chi^{2} \Delta^{2}\right] \cos ^{2} \varphi+2 r t \Delta^{2}\left(a_{2}-a_{1}\right)^{2} \\
& \times\left(1+\exp \left[-\frac{\left(a_{2}-a_{1}\right)^{2} \chi^{2} \Delta^{2}}{2}\right]\left[1-\left(a_{2}\right.\right.\right. \\
& \left.\left.\left.-a_{1}\right)^{2} \chi^{2} \Delta^{2}\right] \cos \varphi\right),
\end{aligned}
$$

and the equality holds up if and only if $\cos \frac{\theta_{i}}{2}=\sin \frac{\theta_{i}}{2}$ and $\cos \frac{\theta_{f}}{2}=\sin \frac{\theta_{f}}{2}$. Here we only consider WMA strategy, $F_{Q}^{\text {tot }}(q)=P_{a} F_{Q}^{a}(q)$. In the weak measurement limit, defined as $\chi \Delta \rightarrow 0, P_{a} F_{Q}^{a}(q)=\left(a_{2}-a_{1}\right)^{2} \Delta^{2}(1+\cos \varphi)$, and the maximum value is $\left(a_{2}-a_{1}\right)^{2} \Delta^{2}$ when $\varphi=0$ or 
$2 \pi$, i.e., $\left|\psi_{i}\right\rangle$ and $\left|\psi_{a}\right\rangle$ are completely orthogonal, but $P_{r} F_{Q}^{r}=0$ and $F_{Q}^{P}=0$. In the strong measurement limit, when $\chi \Delta \gg 1, P_{a} F_{Q}^{a}(q)=\left(a_{2}-a_{1}\right)^{2} \Delta^{2} / 2$, $P_{r} F_{Q}^{r}(q)=\left(a_{2}-a_{1}\right)^{2} \Delta^{2} / 2$, and $F_{Q}^{P}=0$. These results are discussed in the context of imaginary weak values space in weak measurement. It can be seen that when the uncertainty of the pointer state $\Delta$ is fixed, the measurement precision in weak measurement limit is no better than that in strong measurement limit. However, when the parameter $\chi$ is fixed, the measurement precision is always better because using a pointer state with larger $\Delta$ is determined by high temperature. Thus QFIs are all proportional to $\left(a_{2}-a_{1}\right)^{2} \Delta^{2}$.

However, for (24) and (18) we have

$$
\begin{aligned}
& \frac{P_{a} F_{Q}^{a}\left[P_{w m}(q)\right]}{F_{\chi}\left(P_{\text {std }}(p, z=0)\right)} \\
& \leq \frac{\left(a_{2}-a_{1}\right)^{2}}{\left|a_{i}\right|_{\max }^{2}}\left(\frac{1+z}{1-z}\right)\left[-\frac{r^{2} t^{2}}{P_{a}} \exp \left(-k^{2}\right) k^{2} \cos ^{2} \varphi\right. \\
& \left.+\frac{1}{2} r t\left(1+\exp \left(-k^{2} / 2\right)\left(1-k^{2}\right) \cos \varphi\right)\right],
\end{aligned}
$$

where $k^{2}=\left(a_{2}-a_{1}\right)^{2} \chi^{2} \Delta^{2}$, and the equality holds up if and only if $\cos \frac{\theta_{i}}{2}=\sin \frac{\theta_{i}}{2}$ and $\cos \frac{\theta_{f}}{2}=\sin \frac{\theta_{f}}{2}$. The numberator of (25) is QFI for the position's displacement proportional to the imaginary weak values.

Suppose the eigenvalues $a_{1}=1, a_{2}=-1$, implying that $\left|a_{i}\right|_{\max }=1$, and $\theta_{i}=\theta_{f}=\pi / 2$. In weak measurement regime, i.e., $k \ll 1$ and $\varphi \ll 1$, for example, $k^{2}=0.0005$ with $z=0, \varphi=0.05, \frac{P_{a} F_{Q}^{a}\left[P_{w m}(q)\right]}{F_{\chi}\left(P_{\text {std }}(p, z=0)\right)}=$ 0.8327 and $k^{2}=0.0095$ with $z=0, \varphi=0.05$, $\frac{P_{a} F_{Q}^{a}\left[P_{w m}(q)\right]}{F_{\chi}\left(P_{\text {std }}(p, z=0)\right)}=3.9478$. Moreover, when $\varphi=\pi / 2$, $\frac{P_{a} F_{Q}^{a}\left[P_{w m}(q)\right]}{F_{\chi}\left(P_{\text {std }}(p, z=0)\right)}=\frac{1+z}{1-z} / 2$. These results suggest that as $z$ grows, the ratio of (25) can exceed 1 . In other words, by adjusting the temperature $T$, we can give a better estimate of an unknown parameter $\chi$. Therefore, the result breaks the inequality constraint in 31 33

$$
P_{\text {postselection }} F_{\text {weak value }} \leq F_{\text {standard }},
$$

where is the postselection success probability. It is obvious that postselected weak measurement using thermal state pointer, corresponding to the displacement proportional to imaginary weak values, can increase the measurement precision.

\section{Precision measurement in the phase space}

We now consider a scenario that particle-number distribution. The initial state of the quantum system $\left|\psi_{i}\right\rangle$ and one of the quantum pointer $\rho_{t h}(z)$ are both the same as before, while let the observable of the pointer be $\hat{n}$. Thus in the Hamiltonian of Eq. (11) we only use $\hat{n}$ instead of $\hat{q}$, the other is unchanged.
After the interaction $U(t)=e^{-i \chi A n}$ for the initial state of the total system, its time evolution is given by

$$
\rho(z)=e^{-i \chi A n}\left|\psi_{i}\right\rangle\left\langle\psi_{i}\right| \otimes \rho_{t h}(z) e^{i \chi A n} .
$$

Using QFI of the pure state $\left|\lambda_{m}\right\rangle: F_{Q, m}=4\left[\left\langle\lambda_{m}^{\prime} \mid \lambda_{m}^{\prime}\right\rangle-\right.$ $\left.\left|\left\langle\lambda_{m} \mid \lambda_{m}^{\prime}\right\rangle\right|^{2}\right]$ with $\lambda_{m}^{\prime}=\partial \lambda_{m} / \partial \chi,\left|\lambda_{m}^{\prime}\right\rangle=\partial\left|\lambda_{m}\right\rangle / \partial \chi$, and the convexity of the QFI of the mixed state: $Q_{j} \leq$ $\sum_{m} \lambda_{m} F_{Q, m}$, we can obtain QFI of the joint state (27)

$$
Q_{j}=\left(a_{1}-a_{2}\right)^{2}\left(2\langle n\rangle^{2}+\langle n\rangle\right) \sin ^{2} \theta_{i},
$$

where $\langle n\rangle=\frac{z}{1-z} . \quad Q_{j}$ is the maximum amount of information when $\theta_{i}=\pi / 2$, and proportional to quantum scaling $\left(\sim\langle n\rangle^{2}\right)$. From the expression of Eq. (28), it can be seen that $\theta_{i}=0, \pi$ will never provide a better than classical scaling. These correspond to two cases, namely, for the eigenstates of $A:\left|\psi_{i}\right\rangle=\left|a_{1}\right\rangle$ or $\left|a_{2}\right\rangle, P_{a} F_{Q}^{a}=0$, $P_{r} F_{Q}^{r}=0$, and $F_{Q}^{P}=0$. Thus, $F_{Q}^{\text {tot }}=0$.

When the postselected state of the measured system $\left|\psi_{a}\right\rangle=\sum_{i=1}^{2} c_{i}^{\prime}\left|a_{i}\right\rangle$ with $c_{1}^{\prime}=\cos \frac{\theta_{f}}{2}$ and $c_{2}^{\prime}=-\sin \frac{\theta_{f}}{2}$ is made for the total system (27), the reduced state of the pointer is $\rho_{a}^{n}(z)=\sum_{n=0}^{\infty} \Theta_{a}|n\rangle_{m}\left\langle\left. n\right|_{m}\right.$ (unnormalized), where

$$
\begin{aligned}
\Theta_{a} & =(1-z) z^{n}\left[r^{2}+t^{2}-r t\left(e^{i\left[\varphi+\chi\left(a_{1}-a_{2}\right) n\right]}\right.\right. \\
& \left.\left.+e^{-i\left[\varphi+\chi\left(a_{1}-a_{2}\right) n\right]}\right)\right]
\end{aligned}
$$

Using identity $\frac{1}{1-z}=\sum_{n=0}^{\infty} z^{n}$, the probability of successful postselection is

$$
\begin{aligned}
P_{a} & =r^{2}+t^{2}-r t\left[e^{-i \varphi}(1-z) /\left(1-z e^{-i \chi\left(a_{1}-a_{2}\right)}\right)\right. \\
& \left.+e^{i \varphi}(1-z) /\left(1-z e^{i \chi\left(a_{1}-a_{2}\right)}\right)\right] .
\end{aligned}
$$

Thus, we can give the normalized state by

$$
\rho_{a}^{n}(z)=\sum_{n=0}^{\infty} \frac{\Theta_{a}}{P_{a}}|n\rangle_{m}\left\langle\left. n\right|_{m} .\right.
$$

Since $\rho_{a}^{n}(z)$ is a mixed state, and satisfies diagonalization form as $\rho=\sum_{m} \lambda_{m}\left|\lambda_{m}\right\rangle\left\langle\lambda_{m}\right|$, where $\left\{\left|\lambda_{m}\right\rangle\right\}$ forms an orthogonality and complete basis, with $\lambda_{m}$ being the weight of $\left|\lambda_{m}\right\rangle$. According to the well-known formula, the QFI of the mixed state with $\lambda_{m} \neq 0$ is given by (see QFI derivation of [34 36$]$ )

$$
\begin{aligned}
F_{Q} & =\sum_{m} \frac{\left(\lambda_{m}^{\prime}\right)^{2}}{\lambda_{m}}+\sum_{m} \lambda_{m} F_{Q, m}-\sum_{m \neq n} \frac{8 \lambda_{m} \lambda_{n}}{\lambda_{m}+\lambda_{n}} \\
& \times\left|\left\langle\lambda_{m} \mid \lambda_{n}^{\prime}\right\rangle\right|^{2} .
\end{aligned}
$$

The first term is the classical Fisher information for the probability distribution $P(m \mid \chi)=\lambda_{m}(\chi)$. The second term is weighted average over the QFI $F_{Q, m}=$ 
$4\left[\left\langle\lambda_{m}^{\prime} \mid \lambda_{m}^{\prime}\right\rangle-\left|\left\langle\lambda_{m} \mid \lambda_{m}^{\prime}\right\rangle\right|^{2}\right]$ for each pure state in the subset $\left\{\left|\lambda_{m}\right\rangle\right\}$, with $\lambda_{m} \neq 0$. The last term reduces the QFI and hence the estimation precision below the pure state case.

Substitute (31) into the QFI fomula (32), we have

$$
\begin{aligned}
F_{Q}^{a} & =\sum_{n=0}^{\infty}\left(\frac{\Theta_{a}^{\prime 2}}{\Theta_{a} P_{a}}-\frac{2 \Theta_{a}^{\prime} P_{a}^{\prime}}{P_{a}^{2}}+\frac{\Theta_{a} P_{a}^{\prime 2}}{P_{a}^{3}}\right) \\
& =\frac{1}{P_{a}} \sum_{n=0}^{\infty} \frac{\Theta_{a}^{\prime 2}}{\Theta_{a}}-\frac{P_{a}^{\prime^{2}}}{P_{a}^{2}}
\end{aligned}
$$

where $\Theta_{a}^{\prime}=\partial \Theta_{a} / \partial \chi, P_{a}^{\prime}=\partial P_{a} / \partial \chi$, and $P_{a}^{\prime}=\sum_{n=0}^{\infty} \Theta_{a}^{\prime}$. It can be easily seen that the FI in Eq. (33) is not QFI, but is described by the classical Fisher information (CFI) for the probability distribution $\frac{\Theta_{a}}{P_{a}}$ in Eq. (32).

When the postselection fails (with probability $P_{r}=$ $1-P_{a}$ ), namely, the failing postselected state of the measured system $\left|\psi_{r}\right\rangle=\sum_{i=1}^{2} c_{i}^{\prime}\left|a_{i}\right\rangle$ with $c_{1}^{\prime}=\sin \frac{\theta_{f}}{2}$ and $c_{2}^{\prime}=\cos \frac{\theta_{f}}{2}$ is performed for the total system (27), the reduced state of the pointer (normalized) is given by

$$
\rho_{r}^{n}(z)=\sum_{n=0}^{\infty} \frac{\Theta_{r}}{P_{r}}|n\rangle_{m}\left\langle\left. n\right|_{m},\right.
$$

where

$$
\begin{aligned}
\Theta_{r} & =(1-z) z^{n}\left[r^{\prime 2}+t^{\prime 2}+r t\left(e^{i\left[\varphi+\chi\left(a_{1}-a_{2}\right) n\right]}\right.\right. \\
& \left.\left.+e^{-i\left[\varphi+\chi\left(a_{1}-a_{2}\right) n\right]}\right)\right]
\end{aligned}
$$

with $r^{\prime}=\cos \frac{\theta_{i}}{2} \sin \frac{\theta_{f}}{2}$ and $t^{\prime}=\sin \frac{\theta_{i}}{2} \cos \frac{\theta_{f}}{2}$. Note that the failing postselection is not considered in the original scheme and often ignored in experiments. Hence, the CFI of the failing postselection is given by

$$
\begin{aligned}
F_{Q}^{r} & =\sum_{n=0}^{\infty}\left(\frac{\Theta_{r}^{\prime 2}}{\Theta_{r} P_{r}}-\frac{2 \Theta_{r}^{\prime} P_{r}^{\prime}}{P_{r}^{2}}+\frac{\Theta_{r} P_{r}^{\prime^{2}}}{P_{r}^{3}}\right) \\
& =\frac{1}{P_{r}} \sum_{n=0}^{\infty} \frac{\Theta_{r}^{\prime 2}}{\Theta_{r}}-\frac{P_{r}^{\prime^{2}}}{P_{r}^{2}},
\end{aligned}
$$

where $\Theta_{r}^{\prime}=\partial \Theta_{r} / \partial \chi, P_{r}^{\prime}=\partial P_{r} / \partial \chi$, and $P_{r}^{\prime}=\sum_{n=0}^{\infty} \Theta_{r}^{\prime}$.

Obviously, the CFI after the projective measurement is given by [18]

$$
F_{Q}^{P}=\frac{P_{a}^{\prime^{2}}}{P_{a}}+\frac{P_{r}^{\prime^{2}}}{P_{r}},
$$

As $\chi \rightarrow 0, F_{Q}^{P}=\frac{4\left(a_{1}-a_{2}\right)^{2} r^{2} t^{2} \sin ^{2} \varphi}{\left(1-r^{2}-t^{2}+2 r t \cos \varphi\right)\left(r^{2}+t^{2}-2 r t \cos \varphi\right)}\langle n\rangle^{2}$, implying that $F_{Q}^{P}$ can reach the limit of QFI of the joint state $Q_{j}\left(\sim\langle n\rangle^{2}\right)$. Set $\theta_{i}=\theta_{f}=\frac{\pi}{2}$, and we find that

$$
F_{Q}^{P}=\left(a_{1}-a_{2}\right)^{2}\langle n\rangle^{2},
$$

Moreover, in this same situation, the CFIs for both the successful and failed postselection are $P_{a} F_{Q}^{a}=\left(a_{2}-\right.$ $\left.a_{1}\right)^{2} \sin ^{2} \varphi\left(\langle n\rangle^{2}+\langle n\rangle\right) /(2-2 \cos \varphi)$ and $P_{r} F_{Q}^{r}=\left(a_{2}-\right.$ $\left.a_{1}\right)^{2} \sin ^{2} \varphi\left(\langle n\rangle^{2}+\langle n\rangle\right) /(2+2 \cos \varphi)$. This shows that $P_{a} F_{Q}^{a}$ can achieve its maximal amount

$$
P_{a} F_{Q}^{a}=\left(a_{2}-a_{1}\right)^{2}\left(\langle n\rangle^{2}+\langle n\rangle\right)
$$

when $\varphi \rightarrow 0$ or $2 \pi$, i.e., $\left|\psi_{i}\right\rangle$ and $\left|\psi_{a}\right\rangle$ are completely orthogonal, but $P_{r} F_{Q}^{r}=0$. As our calculation shows, whether the successful postselection or the projective measurement (postselected measurement), their FIs indeed scales at the Heisenberg limit $\left(F_{Q} \sim\langle n\rangle^{2}\right)$.

Hence,

$$
\begin{aligned}
F_{Q}^{t o t} & =\sum_{n=0}^{\infty}\left(\frac{\Theta_{d}^{\prime 2}}{\Theta_{d}}+\frac{\Theta_{r}^{\prime 2}}{\Theta_{r}}\right) \\
& \leq 4 r t\left(a_{1}-a_{2}\right)^{2} \frac{z(1+z)}{(1-z)^{2}} \\
& =\left(a_{1}-a_{2}\right)^{2}\left(2\langle n\rangle^{2}+\langle n\rangle\right) \sin \theta_{i} \sin \theta_{f},
\end{aligned}
$$

and the equality holds up if and only if $\cos \frac{\theta_{i}}{2}=\sin \frac{\theta_{i}}{2}$ and $\cos \frac{\theta_{f}}{2}=\sin \frac{\theta_{f}}{2}$. We find that $F_{Q}^{t o t} \leq Q_{j}$, and equality holds up when $\theta_{i}=\theta_{f}$. The maximal $F_{Q}^{t o t}$ and $Q_{j}$ are found for $\theta_{i}=\theta_{f}=\pi / 2$, and equal to $\left(a_{1}-a_{2}\right)^{2}\left(2\langle n\rangle^{2}+\right.$ $\langle n\rangle)$. The results show that the Heisenberg scaling arises in weak measurement using thermal state as a pointer.

\section{CONCLUSION}

In summary, by considering thermal states as the pointers in weak measurement, we have concluded that in the displacement space the QFI corresponding to real weak values is no advantage for the precision of parameter estimation. For imaginary weak values, we have shown that in the case of weak coupling $(\chi \rightarrow 0)$ the maximal QFI after successful postselction can achieve the level of thermal fluctuations $\left(F_{Q} \sim \frac{1+z}{1-z} \sigma^{2}\right)$, and as the temperature increases, QFI is also increased, thereby improving the precision of parameter estimation, in sharp contrast with QFI using Gaussian states (i.e., pure states) as the pointers in the standard strategy which indicates that QFI only achieve the level of zero point fluctuations $\left(F_{Q} \sim \sigma^{2}\right)$.

In the phase space, however, our calculations show that not only successful postselection but postselected measurement itself only contain useful CFI when weak measurement use only classical resources, i.e., thermal states, and in weak measurement limit $(\chi \rightarrow 0)$ their CFI can both attain the Heisenberg scaling $\left(F_{Q} \sim\langle n\rangle^{2},\langle n\rangle=\right.$ $\left.\frac{z}{1-z}\right)$ for the precision of parameter estimation. Obviously, weak measurement using thermal state pointer in phase space can yield calssical-enhanced precision. As the temperature increases, CFI is futher increased. Thus, 
the measurement precision of the Heisenberg limit can be much larger than that of the classical measurement method. It is a known fact that thermal states are easy to be prepared under current experimental conditions. Our work provides a way to realize Heisenberg scaling precision, regardless of utilizing the light or the matter as a pointer.

\section{ACKNOWLEDGMENT}

This work was supported by the Natural Science Foundation of Shaanxi Province (Grant No. 2018JQ1056), the Doctoral Scientific Research Foundation of Yan'an University (Grant No. YDBK2016-04) and Youth Foundation of Yan'an University (Grant No. YDQ2017-09).

[1] R. Schnabel, N. Mavalvala, D. E. McClelland, and P. K. Lam, Quantum metrology for gravitational wave astronomy, Nat. Commun. 1, 121 (2010).

[2] S. L. Danilishin and F. Y. Khalili, Quantum measurement theory in gravitationalwave detectors, Living Rev. Relativ. 15, 5 (2012).

[3] R. X. Adhikari, Gravitational radiation detection with laser interferometry, Rev. Mod. Phys. 86, 121 (2014).

[4] A. Derevianko and H. Katori, Colloquium: physics of optical lattice clocks, Rev. Mod. Phys. 83, 331 (2011).

[5] A. D. Ludlow, M. M. Boyd, J. Ye, E. Peik, and P. O. Schmidt, Optical atomic clocks, Rev. Mod. Phys. 87, 637 (2015).

[6] M. I. Kolobov, The spatial behavior of nonclassical light, Rev. Mod. Phys. 71, 1539 (1999).

[7] L. A. Lugiato, A. Gatti, and E. Brambilla, Quantum imaging, J. Opt. B: Quantum Semiclass. Opt. 4, S176 (2002).

[8] J. P. Dowling and K. P. Seshadreesan, Quantum optical technologies for metrology, sensing, and imaging, J. Lightwave Technol. 33, 2359 (2015).

[9] M. Aspelmeyer, T. J. Kippenberg, and F. Marquardt, Cavity optomechanics, Rev. Mod. Phys. 86, 1391 (2014).

[10] M. A. Taylor and W. P. Bowen, Quantum metrology and its application in biology, Phys. Rep. 615, 1 (2016).

[11] J. J. Bollinger, W. M. Itano, D. J. Winel, and D. J. Heinzen, Optimal frequency measurements with maximally correlated states, Phys. Rev. A 54, R4649 (1996).

[12] P. Walther, J. W. Pan, M. Aspelmeyer, R. Ursin, S. Gasparoni, and A. Zeilinge, De Broglie wavelength of a nonlocal fourphoton state, Nature (London) 429, 158 (2004).

[13] I. Afek, O. Ambar, Y. Silberberg, High-NOON states by mixing quantum and classical light, Science $\mathbf{3 2 8}, 879$ (2010).

[14] K. Goda, O. Miyakawa, E. E. Mikhailov, S. Saraf, et al, A quantum-enhanced prototype gravitational wave detector, Nat. Phys. 4, 472 (2008).

[15] P. Grangier, R. Slusher, B. Yurke, A. LaPorta, Squeezedlight-enhanced polarization interferometer, Phys. Rev. Lett. 59, 2153 (1987).
[16] M. Xiao, L. A. Wu, H. J. Kimble, Precision measurement beyond the shot-noise limit, Phys. Rev. Lett. 59, 278 (1987).

[17] N. Treps, N. Grosse, W. P. Bowen, C. Fabre, H. A. Bachor, P. K. Lam, A quantum laser pointer, Science 301, 940 (2003).

[18] L. J. Zhang, A. Datta, and I. A. Walmsley, Precision Metrology Using Weak Measurements, Phys. Rev. Lett. 114, 210801 (2015).

[19] G. Chen, L. J. Zhang, W. H. Zhang, X. X. Peng, et al, Achieving Heisenberg-Scaling Precision with Projective Measurement on Single Photons, Phys. Rev. Lett. 121, 060506 (2018).

[20] G. Chen, N. Aharon, Y. N. Sun, Z. H. Zhang, et al, Heisenberg-scaling measurement of the single-photon Kerr non-linearity using mixed states, Nature Commun. 9, 1 (2018)

[21] Y. Aharonov, D. Z. Albert, and L. Vaidman, How the result of a measurement of a component of the spin of a spin-1/2 particle can turn out to be 100, Phys. Rev. Lett. 60, 1351 (1988).

[22] G. Li, T. Wang, S. Xu, and H. S. Song, Weak measurement based on thermal noise effect, arXiv: 1507.00850 (2015).

[23] C. Simon and S. E. Polzik, Fock-state view of weakvalue measurements and implementation with photons and atomic ensembles, Phys. Rev. A 83, R040101 (2011).

[24] G. Li, T. Wang, and H. S. Song, Amplification effects in optomechanics via weak measurements, Phys. Rev. A 90, 013827 (2014).

[25] G. Li, L. B. Chen, X. M. Lin, and H. S. Song, Weak measurement amplification in optomechanics via a squeezed coherent state pointer, J. Phys. B: At. Mol. Opt. Phys. 48, 165504 (2015).

[26] G. Li, T. Wang, M. Y. Ye, and H. S. Song, Weak measurement combined with quantum delayed-choice experiment and implementation in optomechanical system, Eur. Phys. J. D 69, 266 (2015).

[27] P. A. Mello and L. M. Johansen, Measurements in Quantum Mechanics and Neumann's Model, AIP Conf. Proc. 1319, 3 (2010).

[28] C. W. Helstrom, Quantum detection and estimation theory (Academic press New York, New York, USA, 1976), Vol. 84.

[29] H. Cramer, Mathematical Methods of Statistics (Princeton University Press, Princeton, NJ, 1946).

[30] M. Cohen, The Fisher information and convexity (Corresp.), IEEE Trans. Inf. Theory 14, 591 (1968).

[31] G. C. Knee and E. M. Gauger, When Amplification with Weak Values Fails to Suppress Technical Noise, Phys. Rev. X 4, 011032 (2014).

[32] G. C. Knee, J. Combes, C. Ferrie, and E. M. Gauger, Weak-value amplification: state of play, Quantum Meas. Quantum Metrol. 3, 32 (2016).

[33] S. Tanaka and N. Yamamoto, Information amplification via postselection: A parameter-estimation perspective, Phys. Rev. A 88, 042116 (2013).

[34] S. Knysh, V. N. Smelyanskiy, and G. A. Durkin, Scaling laws for precision in quantum interferometry and the bifurcation landscape of the optimal state, Phys. Rev. A 83, R021804 (2011).

[35] J. Liu, X. X. Xing, W. Zhong, and X. G. Wang, Quantum Fisher Information for Density Matrices with Arbitrary Ranks, Commun. Theor. Phys. 61, 45 (2014). 
[36] Y. M. Zhang, X. W. Li, W. Yang, and G. R. Jin, Quantum Fisher information of entangled coherent states in the presence of photon loss, Phys. Rev. A 88, 043832

(2013) 